\title{
KV-Verwaltungsgebühren auf Sachkosten
}

— Kapitel V/40 des EBM enthält Pauschalerstattungen für Sachkosten wie z. B. die nach Nr. 40120 EBM berechnungsfähige Kostenpauschale für die Versendung bzw. den Transport von Briefen und/oder schriftlichen Unterlagen bis $20 \mathrm{~g}$ (z. B. im Postdienst Standardbrief) oder für die Übermittlung eines Telefax. Die Pauschale ist in Anlehnung an die Postgebühren mit 0,55 Euro dotiert.

Nach einem aktuellen Urteil des Bundessozialgerichtes (BSG) vom 17.8.2011 (AZ B 6 KA 2/11) können die KVen bei der Berechnung der Verwaltungskostenbeiträge einer Praxis auch diese Erstattungen einbeziehen. Das Urteil bezieht sich zwar konkret auf einen Fall, bei dem sich die KV Niedersachsen gegen eine nephrologische Gemeinschaftspraxis durchsetzen konnte, die ein Dialysezentrum betreibt. Die KV hatte vom Gesamthonorar einschließlich der Pauschalerstattung für Sachkosten bei der Durchführung von Dialysen einen Anteil von 2,2\% für Verwaltungskosten einbehalten. Das Gericht begründete sein Urteil damit, dass im vertragsärztlichen Honorar immer auch Sachkosten enthalten wären.

\section{MMW Kommentar}

Auch wenn das Urteil sehr speziell auf die Erhebung von Verwaltungskosten bei Dialysesachkosten abhebt, kann es auch auf die übrigen Pauschalen im Kapitel V/40 bezogen werden. Die Richter gehen davon aus, dass derartige Pauschalen auch unter dem Aspekt im EBM kalkuliert sind, dass ein günstigerer Einkauf möglich und damit sogar ein Gewinn für die Praxis erzielbar ist. Beim
Kauf einer Briefmarke ist dies allerdings nun eindeutig nicht der Fall, trotzdem fällt auch diese Pauschale unter die Abgabepflicht. Umso wichtiger ist es zu beachten, dass die Leistungsbeschreibung bei der Nr. 40120 EBM wie auch bei vielen anderen Sachkostenerstattungen tatsächlich preisgünstige Alternativen beinhaltet. So kann die „Portopauschale" von 0,55 Euro auch dann erhoben werden, wenn der Brief nicht per Post, sondern per Fax (oder in digitalisierter Form per E-Mail) verschickt wird. In diesem Fall liegen die entstehenden Kosten deutlich niedriger als die Pauschale und selbst unter Berücksichtigung des Verwaltungskostenabzugs entsteht in der Mischkalkulation für die Praxis kein Verlust, sondern ggf. sogar ein kleiner Gewinn.

\section{Finanzierung der neuen Kartenleser gesichert!}

- Kassenärztliche Bundesvereinigung (KBV) und der GKV-Spitzenverband (SpiK) haben sich darauf geeinigt, dass Vertragsärztinnen und -ärzte, die bis zum 30. September 2011 ein Kartenterminal zum Einlesen der elektronischen Gesundheitskarte (eGK) bestellen, grundsätzlich Anspruch auf Refinanzierung der entstandenen Kosten haben. Eine Protokollnotiz in der Änderungsvereinbarung zur Einführung der eGK regelt, dass die Krankenkassen auch bei Lieferung der Geräte nach dem 30. September die Ausstattungskosten übernehmen.

Für die Anschaffung eines stationären Gerätes, das die Daten der eGK lesen kann, erhalten Ärzte 355 Euro. Für ein mobiles Gerät gibt es 280 Euro und für die Installation erstatten die Kassen 215 Euro. Entscheidend ist damit der Bestell- und nicht der Rechnungs- oder Installationstermin. Grund für die neue Regelung sind Lieferprobleme einiger Kartenterminalhersteller.
Die KBV empfiehlt in diesem Zusammenhang, die rechtzeitige Bestellung der Geräte - als Nachweis gegenüber der KV durch den Lieferanten mit Bestelldatum und voraussichtlichem Lieferdatum bestätigen zu lassen.

\section{MMW Kommentar}

So erfreulich diese Nachricht einerseits ist, so problematisch könnte die Umsetzung in der Praxis andererseits werden. Die Ausgabe der eGK durch die Kassen soll nämlich trotzdem wie geplant zum 1. Oktober 2011 erfolgen, sodass bereits ab diesem Zeitpunkt Patienten mit der neuen Gesundheitskarte in die Arztpraxen kommen könnten. Krankenkassen müssen bis Ende dieses Jahres mindestens 10\% ihrer Versicherten mit der elektronischen Gesundheitskarte (eGK) ausstatten. Da mit den neuen Lesegeräten zwar sowohl die alten Krankenversichertenkarten wie auch die eGK gelesen werden können, aber nicht umgekehrt, bleibt in solchen

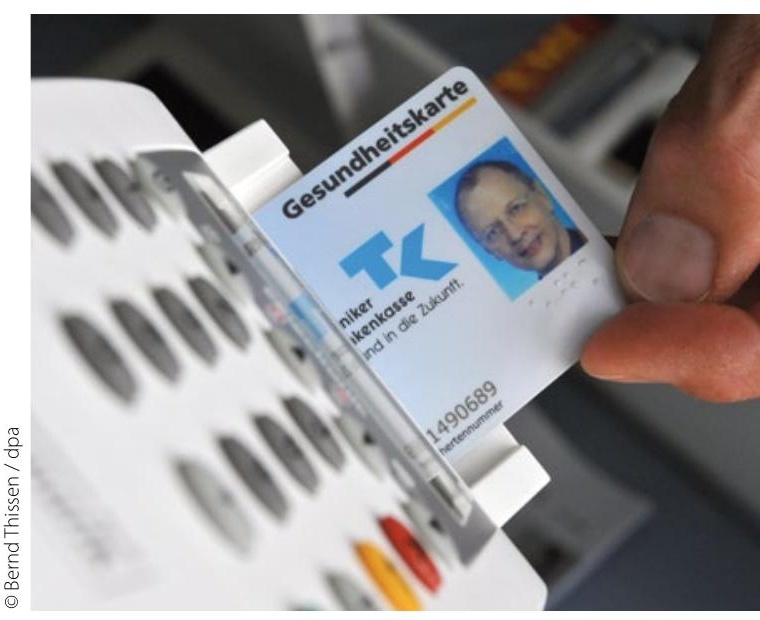

Entscheidend für die Erstattung ist, wann Sie das Lesegerät bestellt haben.

Fällen der Praxis nur die vorübergehende Anwendung des Ersatzverfahrens bei der Patientenerfassung. 